\title{
Fächerübergreifender Unterricht zur Nachhaltigkeit: wissens- oder verhaltensorientiert?
}

\author{
andreas.joppich@gmx.de, Projekt-Agentur Joppich (www.projekte-joppich.de), Berlin \\ **anke.uhlenwinkel@aau.at, Institut für Geographie und Regionalforschung, Alpen-Adria-Universität, Klagenfurt
}

eingereicht am: 21.07.2016, akzeptiert am: 15.12.2016

\begin{abstract}
Fächerübergreifender Unterricht ist eine von Vertretern der Bildung für nachhaltige Entwicklung immer wieder erhobene Forderung. Dabei verstehen sie fächerübergreifend, im Gegensatz zur klassischen auf die Fächer bezogenen Konzeption, oftmals als vornehmlich verhaltensorientierten Unterricht, der weitgehend ohne fachliche Bezüge auskommt. Einen dritten Weg gehen hier sowohl das internationale Projekt „Expedition Grönland“ als auch der neue Berlin-Brandenburger Rahmenlehrplan, in dessen Kontext die Projektergebnisse relevant sind: sie verknüpfen Fächer und politische Bildung im Sinne der Schulgesetze.
\end{abstract}

Keywords: Bildung für Nachhaltige Entwicklung, fächerübergreifender Unterricht, politische Bildung, Grönland

\section{Focusing on Knowledge or Behaviour? - Cross-Disciplinary Curriculum-Making in the Context of Education for Sustainable Development.}

Cross-curricula approaches are one of the main teaching requirements considered important for Education for Sustainable Development. But very often the procedure chosen is quite different from the classical approach as it denies the importance of subjects in favour of behavioural goals. The international project "Expedition Greenland" is following a third approach that links subjects and civic education. The materials developed in the project are compatible to the ideas of the new Berlin-Brandenburg Curriculum.

Keywords: Education for Sustainable Development, cross-curricular approach, citizenship education, civics, Greenland

\section{Die Interdisziplinarität von Nachhaltigkeit}

Einer der Hauptvertreter des Konzeptes der Bildung für nachhaltige Entwicklung in Deutschland ist schon sehr früh davon ausgegangen, dass sein Anliegen ein „interdisziplinäres, fächerübergreifendes Lernen“ (de Haan 2004, 42; vgl. Stoltenberg 2006) voraussetzt. Begründet wird dies damit, dass die „Problemfelder nicht nachhaltiger Entwicklung und Perspektiven zukunftsfähiger Veränderungen [...] heute nicht mehr durch eine Fachwissenschaft oder mit einfachen Handlungsstrategien zu bewältigen“ (ebd., 41) seien, weil sie sich durch komplexe Systemzusammenhänge auszeichneten. Folgte man diesem Argument weiter und nähme man den Terminus fächerübergreifend ernst, ergäbe sich aus diesem Ansatz eine Zusammenführung der Wissensbestände verschiedener Fächer zum besseren Verständnis real vorgefundener Problematiken. Eine solche Zusammenführung scheint aber nicht oder zumindest nicht vorwiegend das Ziel seiner Bildung für nachhaltige Entwicklung, heißt es doch an anderer Stelle, dass der Lerngegenstand gegenüber den zu vermittelnden Handlungskonzepten „zweitrangig“ (ebd.) sei. Dass er trotzdem „nicht gleichgültig“ (ebd.) ist, verdankt er jedenfalls nicht seinen fachlichen Qualitäten, sondern den Vorkenntnissen, Motivationen und Alltagsbezügen der Lernenden (ebd.).

Folgerichtig wird die Interdisziplinarität der Bildung für nachhaltige Entwicklung jenseits des Fachlichen über einen zweiten Zugang legitimiert, die politische Bildung: „Die allgemeine Nachhaltigkeitsdebatte legt es ebenso wie die Ausdifferenzierung des Kompetenzkonzeptes nahe, Bildung für eine nachhaltige Entwicklung explizit als politische Bildung zu verstehen“ (ebd., 42). Dieser Schluss erscheint auch insoweit logisch, als das Konzept der Nachhaltigkeit im politischen Kontext entstanden ist und insbesondere die Sozialwissenschaft eine Beschäftigung mit 
dem Thema deswegen lange verweigert hat (Melde 2012, 22; vgl. Miossec et al. 2004).

Nichtsdestotrotz wird von de Haan aber auch hier gleich wieder Abstand genommen zum Fach der Politischen Bildung: „Die politischen Dimensionen der Nachhaltigkeitsthematik werden allerdings in der Regel nicht von Seiten der politischen Bildung bearbeitet" (ebd., 44). Die hier artikulierte geringe Fachgebundenheit der politischen Bildung verdankt sich dem dieser Variante der Bildung für nachhaltige Entwicklung zugrundeliegenden Demokratieverständnis. Im Kontext der hier angestrebten Bildung für nachhaltige Entwicklung steht demnach nicht Demokratie als Herrschaftsform im Mittelpunkt des Interesses, sondern Demokratie als alltägliche Lebensform. Für den Politikunterricht charakteristische Themen wie der Rechtsstaat, Wahlen oder soziale Sicherungssysteme sind in dieser Perspektive somit „eher nachrangig“ (ebd., 43). Dem Verständnis der nachhaltigen Entwicklung als „normativer Handlungsrahmen" (ebd., 39) gemäß (vgl. Miossec et al. 2004) rücken stattdessen ethische Begrifflichkeiten wie Toleranz, Solidarität und Chancengleichheit in den Vordergrund, die in jedem Fach unterrichtet werden können. Entsprechend wird im österreichischen „Leitfaden zur neuen Reifeprüfung in Geographie und Wirtschaftskunde" (BMUKK 2012, 100) die Vorbereitung der Lernenden auf zukünftige Entwicklungen auch „von den Leitgedanken der Nachhaltigkeit und der Solidarität geprägt" (ebd., 102). Gezielt wird somit auf einen „Mentalitätswandel“ (de Haan 2004, 40) und eine „weitreichende Modifikation in den Lebensweisen der Menschen" (ebd.; vgl. Lauströer 2008). Diese Zwecksetzung schränkt einen rationalen Diskurs insoweit ein, als sie Fakten und Argumente, die dem Zweck des Mentalitätswandels nicht dienlich sind, möglichst ungesagt und damit unberücksichtigt lässt (vgl. Wulf 2006) ${ }^{1}$.

Dieser Interpretation stehen auch mit der Bildung für nachhaltige Entwicklung assoziierte Denkfiguren wie etwa der Dreiklang von Wissen, Einstellungen und Handeln (vgl. Jekel 2008) oder der Syndromansatz (vgl. Schindler 2005; Lauströer 2008) nicht entgegen. Der Dreiklang von Wissen, Einstellung und Handeln kann empirisch nicht belegt werden. Gerade auch sehr umweltbewusste Menschen, verhalten sich oftmals nicht entsprechend (vgl. Kuckartz 1998, 44). Der Syndromansatz ist, ebenso wie das Konzept der Nachhaltigkeit, im politischen Kontext entstanden (vgl. Schindler 2005, 47). Dieser Ansatz ist in der akademischen Geographie frühzeitig aufgrund seiner „wenig analytischen und dafür umso stärker tautologischen Form des ,Teufelskreises “ (Krings 2002, 131) kritisiert worden. Dies führe zu einer Vernachlässigung gesellschaftlicher Ungleichheiten und damit zu einer Ausklammerung der Machfrage (ebd.), ohne die gesellschaftliche Entwicklungen nicht erklärt werden können. Ebenfalls missachtet wird die Tatsache, dass im Kontext der Soziologie ein Modell zur Erklärung gesellschaftlicher Funktionssysteme entwickelt wurde, das explizit von einem „Kappen von determinierenden Umwelt-
Mit der Zurückstellung fachlicher Anschlussfähigkeit navigiert sich die Bildung für nachhaltige Entwicklung allerdings in eine überaus fragwürdige Position, denn wo die sich in Fächern artikulierenden unverfügbaren Kenntnisse für nachrangig erklärt werden, gibt es auch keine ausreichende „objektive Außenseite demokratischer Verfahren" (Möllers 2009, 45) mehr, an der sich gesellschaftliche Veränderungen korrigieren lassen könnten. Infrage gestellt wäre damit insbesondere das kritische Denken, das für jede Form der Selbstreflektion essentiell ist (vgl. Butler 2007) und auch als Ziel kompetenzorientierter Bildung für nachhaltige Entwicklung deklariert wird.

Im Folgenden soll am Beispiel eines internationalen und interdisziplinären Projekts zur Frage der nachhaltigen Entwicklung in Grönland zu Zeiten der Wikinger und heute ein Ausweg aus der sich hier abzeichnenden Sackgasse demokratischer Bildung gezeigt werden. Zu diesem Zweck wird zunächst eine kurze theoretische Standortbestimmung des fächerübergreifenden Unterrichts im Kontext schulischer Bildung vorgenommen, um daran anschließend organisatorische und curriculare Umsetzungen unter besonderer Berücksichtigung des neuen Berlin-Brandenburger Rahmenlehrplans diskutieren zu können und zu zeigen, wie die Ergebnisse des Grönland-Projekts sinnvoll in diesen Kontext integriert werden können. Dabei wird die praktische Umsetzung auch dahingehend reflektiert, wie sie auf die theoretischen Vorstellungen zurückwirkt oder jedenfalls wirken könnte.

\section{Fächerübergreifender Unterricht: eine theoretische Standortbestimmung}

Fächerübergreifenden Unterricht hat es bereits lange vor der Erfindung und Popularisierung des Konzepts der Bildung für nachhaltige Entwicklung gegeben (vgl. Huber \& Effe-Stumpf 1994). Für Hilbert Meyer ist er ,ein ganzheitlicher, zumeist handlungsorientiert gestalteter Unterricht, in dem fachliche Ansprüche, Wissensbestände und Prinzipien mehrerer Fächer herangezogen werden, um die den Schülern gestellten Lernaufgaben zu bewältigen" (Meyer 2007, 6). Diese klassische Definition kombiniert zwei Bestandteile, die in der Praxis oftmals je nach Schüler/innenschaft und Jahrgangsstufe unterschiedlich betont werden: Der ganzheitliche, handlungsorientierte Anspruch findet sich zumeist in jüngeren Klassen und Schulformen außerhalb des Gymnasiums, der Anspruch der problemlösenden Nutzung von Wissensbeständen und Prinzipien dagegen in höheren Jahrgangsstu-

einflüssen“ (Luhmann 2001, 14) ausgeht, um den „Überschuss an Möglichkeiten, der für das System selbst unkalkulierbar ist“ (ebd.) fassen zu können. 
fen und im Gymnasium (vgl. Huber \& Effe-Stumpf 1994). Trotz dieser unterschiedlichen Schwerpunkte liegt beiden Formen ein Verständnis von Fächern als „theoretische Konstrukte zur Kodifizierung schulisch relevanten Fachwissens" (Meyer 2007, 5) zugrunde, sei es als Ziel der kindlichen schulischen Entwicklung oder als Voraussetzung fächerübergreifender Projekte.

In jüngerer Zeit ist diese Vorstellung fächerübergreifenden Unterrichts in ein anderes Anliegen transformiert worden. Nun geht es nicht mehr darum, neben der fachlichen Systematik, eine allgemeines Wissenschaftsverständnis oder ein ganzheitliches Weltbild zu vermitteln (Huber \& Effe-Stumpf 1994), sondern darum, „neue Fächer oder zumindest wesentliche Aspekte neuer Fächer in den Schulunterricht zu integrieren" (Schnack 2011, 8), ohne dass der langwierige Weg der Veränderung der Stundentafel gegangen werden muss (vgl. ebd.). Das österreichische Beispiel des „GW“ bietet hierfür einen frühen Prototyp. Zu den heutigen neuen „Fächern“ werden neben Informatik und Recht, die immerhin eine Entsprechung im kodifizierten akademischen Fachwissen haben, auch Medien- und Verbraucherbildung gezählt (ebd.). In eine den letztgenannten Beispielen inhärente verhaltensorientierte Konzeptualisierung von fächerübergreifendem Unterricht ließe sich ohne weiteres auch der oben genannte Ansatz der Bildung für nachhaltige Entwicklung als Vermittlung der Demokratie als Lebensform integrieren. Allerdings ist mit der damit implizierten rationalen Inhaltsleere des fächerübergreifenden Unterrichts auch „die Gefahr der Beliebigkeit [...] gegeben, da nun einmal das Korsett eines Faches fehl"“ (Huber \& Effe-Stumpf 1994, 80).

Beide Konzepte nehmen im Rahmen verschiedener Theorieansätze zur Beschreibung von Unterricht unterschiedliche Positionen ein. Legt man die Vorstellung zugrunde, dass Lehr- und Unterrichtplanung versuchen, eine Balance zwischen den drei Basiskategorien Fachwissen, gesellschaftliche Ziele und pädagogische Prozesse herzustellen (vgl. Marsden 1997), dann wäre die klassische Form des fächerübergreifenden Unterrichts eher der Kategorie des Fachwissens und die verhaltensorientierte Form eher den gesellschaftlichen Zielen zuzuordnen. Eine solche Zuordnung legt allerdings nahe, dass es sich bei der zweiten Variante nicht um einen fächerübergreifenden Unterricht handelt, sondern um normierenden Unterricht, der im Zweifelsfalle meint, ohne die systematischen Inhalte der etablierten, auf einer langen Forschungstradition beruhenden Fächer auskommen zu können und so schnell subjektiv und präskriptiv wird.

Fachwissen wird bei Marsden nicht als eine Ansammlung von Fakten verstanden, sondern als ein konzeptuelles Fachverständnis. Damit impliziert er im Sinne von Young und Muller ein Lehr- und
Unterrichtskonzept der dritten Generation (F3). In dem theoretischen Rahmen ihrer Bildungsszenarien entspricht ein F1-Konzept einem an der reinen Inhaltsvermittlung orientierten Unterricht, in dessen Kontext die vorgegebenen Fächer als starre Struktur betrachtet werden, die sich die Lernenden anzueignen haben. Ein F2-Konzept orientiert sich dagegen vor allem an der Vermittlung von fachübergreifenden Kompetenzen, die mit Vorstellungen des "Lernen lernens“ verbunden sind und bei deren Aneignung Inhalte als zweitrangig betrachtet werden. Im Kontext des F3Konzepts werden das Wissen-Was und das WissenWie zu einem am Denken wissenschaftlicher Disziplinen orientierten Unterricht zusammengeführt (vgl. Young \& Muller 2010). Das F1-Konzept sehen Young und Muller dabei als eine Vermittlung von knowledge of the powerful, mit dessen Hilfe die gebildeten Schichten ihre Position festigen, das F2-Konzept als eine Form, mit der versucht werden sollte, diese Dominanz aufzubrechen, durch die sie aber letztlich nur in einer subtileren Form fortgeführt wurde. Mit dem F3Konzept soll diesem Problem begegnet werden, indem allen Lernenden die Chance gegeben wird, powerful knowledge zu erwerben, d. h. ein Wissen, das es ihnen erlaubt, die Welt nach ihren begründeten Vorstellungen und im gesellschaftlichen Kontext zu gestalten. In dieser Kategorisierung entspricht die klassische Form des fächerübergreifenden Unterrichts abhängig vom jeweiligen Fachverständnis entweder dem F1- oder dem F3-Konzept. In Deutschland kann man beides erkennen, wobei das F3-Konzept eher Modellversuchen wie etwa dem Oberstufen-Kolleg zuzuordnen wäre (Huber \& Effe-Stumpf 1994), während das F1-Konzept sich häufig in den Regelschulen findet. Die verhaltensorientierte Form des fächerübergreifenden Unterrichts würde aufgrund des Mangels an inhaltlicher Substanz dagegen eher dem F2-Konzept zugeordnet werden. Hier steht allerdings in Frage, ob es sich beim verhaltensorientierten Ansatz überhaupt um einen fächerübergreifenden Ansatz handeln kann oder ob dieser Ansatz nicht aufgrund der Negation der Relevanz der Fächer im F2-Konzept eher einen ungefächerten Zugang darstellt.

Fachübergreifender Unterricht kann dort, wo er tatsächlich durchgeführt wird, sehr unterschiedlich organisiert sein. Am deutlichsten über die ursprünglichen Fachgrenzen hinaus gehen Formen des fächeraussetzenden und fächerergänzenden Unterrichts, da sie Zeit in den Unterrichtswochen beanspruchen, die ansonsten von den Fächern genutzt würde: Im ersten Fall in Form von Projektwochen, im zweiten Fall in ausgewiesenen Stunden für nicht an Fachsystematiken gebundenen Unterricht in der regulären Stundentafel (vgl. ebd.). Dagegen lassen sich fächerüberschreitender und fächerverknüpfender Unterricht 
allein durch das Engagement einzelner Lehrkräfte umsetzen, indem im Falle des fächerüberschreitenden Unterrichts eine Lehrkraft Inhalte integriert, die über die Grenzen des eigenen Faches hinausgehen und im Falle des fächerverknüpfenden Unterrichts zwei Lehrkräfte mittels Absprachen aufeinander abgestimmte Fachinhalte behandeln (vgl. Labudde 2006; Huber \& Effe-Stumpf 1994). Zwischen diesen beiden Extremen ist der fächerkoordinierende Unterricht angesiedelt (vgl. Huber \& Effe-Stumpf 1994). Im fächerkoordinierenden Unterricht bleiben die Fächer zwar bestehen und werden von den jeweiligen Fachlehrkräften unterrichtet, ihre Inhalte werden aber bereits in der übergreifenden curricularen Planung aufeinander bezogen (vgl. ebd.). Dieser Bezug wird regelmäßig durch übergeordnete Themen, etwa mit Rückgriff auf die klafkischen Schlüsselprobleme (vgl. Klafki 1996), hergestellt (vgl. Labudde 2006).

Als Resümee aus diesen theoretischen Überlegungen lässt sich nur schließen, dass Bildung für nachhaltige Entwicklung in ihrer oben dargelegten Konzeptualisierung weniger als fächerüberschreitend, fächerverknüpfend oder fächerkoordinierend, als vielmehr als ein übergreifendes Erziehungsziel im Sinne der föderalen Schulgesetze zu verstehen ist, das unter anderem auch im Kontext eines fächeraussetzenden oder fächerergänzenden Unterrichts verfolgt werden kann. Jüngere curriculare Vorgaben überwinden die Unausgewogenheit zwischen der Gewichtung der politischen Zielvorgaben und den fachlichen Inhalten, indem sie beide Teile sinnvoll aufeinander beziehen. Dies wird im folgenden Abschnitt zu zeigen sein.

\section{Die organisatorische Integration fächer- übergreifenden Unterrichts im Kon- text des neuen Berlin-Brandenburger Rahmenplans}

Der neue Berlin-Brandenburger Rahmenlehrplan besteht aus drei Teilen. In Teil A werden allgemeine Grundsätze des Lehrens und Lernens beschrieben (vgl. SBJW \& MBJS 2015a). Teil B widmet sich den fächerübergreifenden Kompetenzen, die zum einen allgemeine Fertigkeiten wie die Sprach- und Medienbildung umfassen und zum anderen übergreifende Themen benennen, die einen deutlichen Rückbezug auf die Erziehungsziele der Schulgesetze erkennen lassen. $\mathrm{Zu}$ diesen Themen gehört neben der oben bereits angesprochenen Verbraucherbildung auch die Bildung für nachhaltige Entwicklung (vgl. SBJW \& MBJS 2015b). Teil C des Rahmenlehrplans ist fachgebunden und beschreibt Themen und Inhalte ebenso wie die im Fach zu erreichenden Kompetenzen und Standards (vgl. SBJW \& MBJS 2015c, d und e; vgl. Abb. 1).

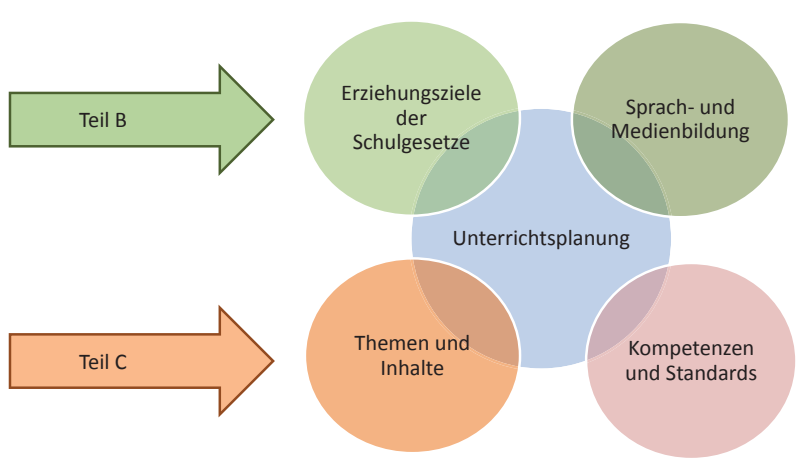

Abb. 1: Zusammenwirken der Teile B und C des Berlin-Brandenburger Rahmenlehrplans (Quelle: Eigene Darstellung).

Im Teil C des Berlin-Brandenburger Rahmenlehrplans ist für die gesellschaftswissenschaftlichen Fächer in den Klassen 7 bis 10 ein fächerkoordinierender Unterricht zu insgesamt vier übergreifenden Themen vorgesehen. Neben den in Tabelle 1 dargestellten Themen „Migration und Bevölkerung“, „Armut und Reichtum“ sowie „Konflikte und Konfliktlösungen“, die hier ausführlich beschrieben werden, weil sich die Unterrichtsvorschläge zu Grönland auf diese Themenfelder beziehen, wird auch das Thema „Europa im Wandel“ im Fächerverbund unterrichtet (vgl. SBJW \& MBJS 2015c, d und e).

Die Anliegen der Bildung für nachhaltige Entwicklung werden über die im Teil B des Rahmenlehrplans dargestellten, als „übergreifende Themen “ bezeichneten Ziele in den Unterricht integriert (vgl. SBJW \& MBJS 2015b), die sich jedenfalls in Teilen aus den Bildungs- und Erziehungszielen der föderalen Schulgesetze der beiden beteiligten Bundesländer ableiten lassen. Dabei fällt die Herleitung der Bildung für nachhaltige Entwicklung aus dem Berliner Schulgesetz eindeutiger aus, heißt es dort doch, dass Schülerinnen und Schüler befähigt werden sollen, „die Auswirkungen des eigenen und gesellschaftlichen Handelns auf die natürlichen lokalen und globalen Lebensgrundlagen zu erkennen, für ihren Schutz Mitverantwortung zu übernehmen und sie für die folgenden Generationen zu erhalten" ( $\$ 3$ Abs. 3 S. 5 SchulG). In Brandenburg dagegen lässt sich das Anliegen nur über das deutlich individueller orientierte Ziel rechtfertigen, Schülerinnen und Schüler in ihrer Fähigkeit und Bereitschaft zu fördern, „ihre Verantwortung für die eigene Gesundheit, für den Erhalt der Umwelt und die Sicherung der natürlichen Lebensgrundlagen zu begreifen und wahrzunehmen" (\$ 4 Abs. 5 S. 13 BbgSchulG).

Trotz ihrer etwas irreführenden Benennung entsprechen die übergreifenden Themen des Rahmenlehrplans Marsdens gesellschaftlichen Zielen, da sie im Verständnis des Lehrplans, aber auch im Sinne der klafkischen Schlüsselprobleme, „besonders bedeutsame gesellschaftliche Herausforderungen" (SBJW \& 
Tab.1: Module im gesellschaftswissenschaftlichen Fächerverbund (Quelle: eigene Zusammenstellung aus SBJW \& MBJS 2015a, b und c)

\begin{tabular}{|c|c|c|c|}
\hline $\begin{array}{l}\text { Fach } \\
\text { Themenfeld }\end{array}$ & Geschichte & Geographie & Politische Bildung \\
\hline $\begin{array}{l}\text { Migration } \\
\text { und Bevölke- } \\
\text { rung }\end{array}$ & $\begin{array}{l}\text { Leitfrage (z. B. Verlust, Her- } \\
\text { ausforderung, Katastrophe?) } \\
\text { - Ostsiedlung und Binnenko- } \\
\text { lonisation } \\
\text { - Frühneuzeitliche (Zwangs-) } \\
\text { Migration (z. B. Hugenotten, } \\
\text { Böhmen, Türk/innen, Afrika- } \\
\text { ner/innen) nach Berlin und } \\
\text { Brandenburg } \\
\text { - Migration im 19. Jahrhun- } \\
\text { dert nach Amerika, Ver- } \\
\text { schleppung der afrikani- } \\
\text { schen und Vertreibung der } \\
\text { indigenen Bevölkerung }\end{array}$ & $\begin{array}{l}\text { Demographische Entwicklung } \\
\text { in regionaler und globaler } \\
\text { Dimension } \\
\text { - Bevölkerungswachstum } \\
\text { - Bevölkerungszusammenset- } \\
\text { zung nach Alter, Geschlecht } \\
\text { - Schrumpfende und alternde } \\
\text { Bevölkerung als Herausfor- } \\
\text { derung } \\
\text { - Migration } \\
\text { Verstädterung } \\
\text { - Ursachen und Folgen für } \\
\text { städtische und/oder ländli- } \\
\text { che Räume } \\
\text { - Landflucht } \\
\text { - Stadt als Entwicklungsmotor }\end{array}$ & $\begin{array}{l}\text { Integration und Teilhabe } \\
\text { - universelle Menschenrechte } \\
\text { - Asylrecht in Deutschland und Europa } \\
\text { - Migrationspolitik in Deutsch- } \\
\text { land, Leben von Flüchtlingen in } \\
\text { Deutschland und Europa } \\
\text { Migrationsursachen } \\
\text { - Push- und Pull-Faktoren, z. B. Ar- } \\
\text { mut, Kriege, Wohlstand, Sicher- } \\
\text { heit und Freiheit } \\
\text { - Fallbeispiele: Zu- und Abwande- } \\
\text { rung in Berlin und Brandenburg, } \\
\text { Transnationalismus } \\
\text { Biographien und hybride Identitäten } \\
\text { - Integration, Transnationalismus, } \\
\text { Marginalisierung und Segregation } \\
\text { - Fallbeispiele-Einwanderung und Le- } \\
\text { ben in einer vielfältigen Gesellschaft: } \\
\text { Kultur, Ethnie, Geschlecht, sexuelle } \\
\text { Orientierung, Weltanschauung, etc. }\end{array}$ \\
\hline $\begin{array}{l}\text { Armut und } \\
\text { Reichtum }\end{array}$ & $\begin{array}{l}\text { Leitfrage (z. B. Hilfe bei indi- } \\
\text { vidueller Armut-eine Verant- } \\
\text { wortung der Allgemeinheit?) } \\
\text { - Mittelalter: Armut, Reichtum } \\
\text { und Christentum } \\
\text { - Frühe Neuzeit: Armenpolitik } \\
\text { städtischer Eliten zwischen } \\
\text { Fürsorge und Repression } \\
\text { - Industriezeitalter: Soziale Fra- } \\
\text { ge - Antworten von Wirt- } \\
\text { schaft, Religion und Politik }\end{array}$ & $\begin{array}{l}\text { Leben in der Einen Welt/ } \\
\text { Entwicklungsunterschiede } \\
\text { - Hunger und Überfluss } \\
\text { - Ursachen / Folgen von Armut } \\
\text { - Wege aus der Armut } \\
\text { - fairer Handel } \\
\text { - Entwicklungsindikatoren }\end{array}$ & $\begin{array}{l}\text { Soziale Ungleichheit } \\
\text { - Dimensionen: Status, Einkommen, } \\
\text { Bildung, Beruf, Herkunft/Milieu, } \\
\text { Geschlecht, sexuelle Orientierung, } \\
\text { Alter, Behinderung } \\
\text { - Lebensformen und -situationen, } \\
\text { Sozialisation } \\
\text { - Armut (Definitionen), soziale Ge- } \\
\text { rechtigkeit } \\
\text { - Sozialstruktur und deren Be- } \\
\text { sonderheit in der Region/Stadt } \\
\text { Teilhabechancen } \\
\text { - Mitwirkungsmöglichkeiten von } \\
\text { Kindern und Jugendlichen } \\
\text { - Bildungsbiographien } \\
\text { Sozialstaat } \\
\text { - Institutionen und Einrichtungen } \\
\text { (insbesondere ihre Zuständigkei- } \\
\text { ten für Kinder und Jugendliche) } \\
\text { - soziale Leistungen (insbesondere } \\
\text { für Kinder und Jugendliche) }\end{array}$ \\
\hline $\begin{array}{l}\text { Konflikte } \\
\text { und Konflikt- } \\
\text { lösungen* } \\
\text { * Das Thema wurde } \\
\text { aufgrund von Kritik des } \\
\text { VDSG LV Berlin an der } \\
\text { Anhörungsfassung in der } \\
\text { offiziellen Endfassung des } \\
\text { Rahmenplans Geographie } \\
\text { in „"llimawandel und Kli- } \\
\text { maschutz als Beispiel für } \\
\text { internationale Konflikte } \\
\text { und Konfliktlösungen" } \\
\text { umformuliert. In den } \\
\text { anderen beiden Fächern } \\
\text { blieb es bestehen. }\end{array}$ & $\begin{array}{l}\text { Leitfrage (z. B. Viele Pläne - } \\
\text { noch - keine Lösung?) } \\
\text { - z. B. Nahost-Konflikt }\end{array}$ & $\begin{array}{l}\text { - Ursachen und regionale/ } \\
\text { globale Folgen des Klima- } \\
\text { wandels } \\
\text { - Nachhaltige Maßnahmen } \\
\text { des Klimaschutzes } \\
\text { - Interessenkonflikte beim Kli- } \\
\text { maschutz }\end{array}$ & $\begin{array}{l}\text { Kriege, Konflikte, Terrorismus } \\
\text { - Definitionen } \\
\text { - Konfliktanalyse am Fallbeispiel: } \\
\text { Gegenstand/Interessen, Ursachen, } \\
\text { Akteure, Lösungsstrategien (z. B. In- } \\
\text { strumente der zivilen Konfliktbearbei- } \\
\text { tung), internationale Dimension, etc. } \\
\text { - Failed States } \\
\text { Entwicklungspolitik und Friedens- } \\
\text { sicherung } \\
\text { - internationaleAkteure(z. B.UN,NGOs, } \\
\text { EU,NATO): SelbstverständnisundZiele } \\
\text { - Fallbeispiele } \\
\text { - Rolle Deutschlands }\end{array}$ \\
\hline
\end{tabular}


MBJS 2015a, 5) abbilden. Gerade auch in Bezug auf die der Bildung für nachhaltige Entwicklung zuzuordnenden Anteile sollen in diesem Zusammenhang insbesondere die unterschiedlichen fachlichen Sichtweisen thematisiert werden: „Zielkonflikte zwischen den Entwicklungsdimensionen bieten die Chancen, besonders in den gesellschaftswissenschaftlichen und naturwissenschaftlichen Fächern problemorientiert aufgegriffen zu werden und ermöglichen es, dass die Lernenden sich mit den gegenwärtigen und zukünftigen Problemen und Herausforderungen auseinandersetzen" (SBJW \& MBJS 2015b, 34). Professionell umgesetzt, lässt dieser Rahmenlehrplan somit auch die Förderung von powerful knowledge im Sinne von Youngs und Mullers F3-Konzept zu (vgl. Uhlenwinkel 2015). Letztendlich kommt es darauf an, ob der einzelne Lehrende es versteht, die Teile B und C des Rahmenlehrplans im Bewusstsein seiner Verantwortung für den individuellen Schüler und die individuelle Schülerin wie für die gesellschaftliche Entwicklung sinnvoll aufeinander zu beziehen.

\section{Ein fachlich orientierter fächerübergrei- fender Unterricht am Beispiel Grönland}

Im Kontext des internationalen Projekts „Expedition Grönland“ ist ein Unterrichtsvorschlag entstanden, der die Vorstellungen des Rahmenlehrplans mit Leben füllen und somit illustrieren kann.

Ausgangspunkt des Projektes war die Beschäftigung mit den Siedlungsaktivitäten der Wikinger auf Grönland unter dem Gesichtspunkt der Nachhaltigkeit. Dabei wird auf neuere Forschungen zurückgegriffen, die das Bild der „Halsstarrigkeit“ (Behringer 2011, 136) der Wikinger, die „hartnäckig an ihrer bäuerlichen Lebensweise" (ebd.) festhielten, zumindest relativieren und somit auch dem Schluss widersprechen, dass die Wikinger sich anders als die Inuit durch einen „Mangel an Anpassung" (ebd.) an den damaligen Klimawandel auszeichneten, was letztlich zu ihrem Untergang geführt habe. Stattdessen wird davon ausgegangen, dass die sich durch Investitionen in die soziale und materielle Infrastruktur sowie den Aufbau eines florierenden Außenhandels auszeichnende gesellschaftliche Entwicklung zwar über einen langen Zeitraum überaus effektive Adaptation an Veränderungen erlaubt hat (vgl. Berglund 2010), dann aber aufgrund ihrer Pfadabhängigkeit angesichts multipler gesellschaftlicher und natürlicher Herausforderungen an ihre Grenzen stieß (vgl. Dugmore et al. o. J.). Bereits in der ersten Siedlungsphase sind die grönländischen Wikinger danach dazu übergegangen, die Erträge aus der Landwirtschaft durch eine gemeinschaftlich organisierte Robbenjagd im Frühjahr zu ergänzen, um so annuell wiederkehren- de Lücken in der Versorgung mit Nahrungsmitteln zu schließen (vgl. National Museum of Denmark 2008; Arneborg et al. 2012). Ebenso gemeinschaftlich organisiert war die sommerliche Walrossjagd im Norðursetur, einem Jagdgebiet nördlich der beiden Hauptsiedlungsgebiete (vgl. Dugmore et al. 2012; Dugmore et al. o. J.). Das hier erbeutete Elfenbein war in Europa ebenso gefragt wie die Walrosshaut, aus der man Seile fertigte. Diese Jagd diente somit vor allem dem Handel und der dadurch möglichen Beschaffung von Gütern, die nicht selbst hergestellt werden konnten (vgl. Frei et al. 2015). Da beide Jagdformen Kooperation voraussetzten, bildete sich ein stratifiziertes Gemeinwesen mitsamt den dazugehörigen Rechtsgrundlagen heraus (vgl. Berglund 2010; Madsen 2015). Letzteres war offenkundig geeignet die Übernutzung der Karibu-Bestände zu verhindern, eine Leistung, die der egalitären Inuit-Gesellschaft nicht gelang (vgl. Dugmore et al. 2012; Dugmore et al. o. J.). Warum dieses System zusammengebrochen ist, kann bisher nicht abschließend erklärt werden (vgl. Stockinger 2013). Vermutlich kamen eine Reihe unterschiedlich schnell ablaufender Veränderungen zusammen: Neben der Klimaabkühlung vor allem der Einbruch des Handels mit Europa aufgrund von Nachfrageverschiebungen (vgl. Frei et al. 2015, Dugmore et al. 2012; Dugmore et al. o. J.) und der Ausbruch der Pest (vgl. Dugmore et al. 2012; Dugmore et al. o. J.), die damit verbundene Einstellung des Schiffverkehrs nach Island und Norwegen (vgl. Stockinger 2013), die Abwanderung von Einwohner/ innen aus Grönland selbst, wodurch die Ausübung gemeinschaftlicher Tätigkeiten erschwert wurde (vgl. Dugmore et al. 2012; Dugmore et al. o. J.), und die Konkurrenz der einwandernden Inuit (vgl. Stolz 1991; Walk 2002).

Diese Erkenntnisse werden im Projekt genutzt, um Folgen des heutigen Klimawandels in Grönland verstehen zu können und vor allem zu beurteilen, „inwieweit Gesellschaften überhaupt in der Lage sind, eine solche umfassende und weitreichende Transformation zu bewältigen, wie sie das Konzept der zukunftsfähigen Entwicklung impliziert" (Kopfmüller et al. 2001, zit. nach de Haan 2004, 40). Im Bereich der politischen Bildung können sie helfen zu reflektieren, inwieweit es möglich ist, „die Zukunft als offen und gestaltbar aufzufassen" (de Haan 2004, 41) sowie ob und, wenn ja, bis zu welchem Grade „durch vorausschauendes Denken und Handeln [...] mögliche Entwicklungen für die Zukunft bedacht sowie Chancen und Risiken von aktuellen und zukünftigen, auch unerwarteten Entwicklungen thematisiert werden" (ebd.) können. Damit sollen die Bemühungen um nachhaltige Entwicklung nicht grundsätzlich infrage gestellt werden, es soll aber sowohl einer normativen Überforderung der Lernenden und Lehrenden vorgebeugt als auch 
Tab.2: Die Nachhaltigkeit der Entwicklung Grönlands als Thema für die Module im gesellschaftswissenschaftlichen Fächerverbund (eigene Darstellung)

\begin{tabular}{|c|c|c|c|}
\hline $\begin{array}{l}\text { Fach } \\
\text { Themenfeld }\end{array}$ & Geschichte & Geographie & Politische Bildung \\
\hline \multirow[t]{2}{*}{$\begin{array}{l}\text { Migration } \\
\text { und Bevölke- } \\
\text { rung }\end{array}$} & $\begin{array}{l}\text { - Besiedlung Grönlands } \\
\text { - Binnenmigration als Folge } \\
\text { des Klimawandels }\end{array}$ & $\begin{array}{l}\text { - Bevölkerungsentwicklung im } \\
\text { heutigen Grönland } \\
\text { - Binnenmigration als Folge } \\
\text { des Klimawandels und poli- } \\
\text { tische Entscheidungen }\end{array}$ & $\begin{array}{l}\text { - Diskussion über Arbeitsmigration } \\
\text { für den Bergbau (seltene Erden) } \\
\text { - Migration im eigenen Umfeld }\end{array}$ \\
\hline & \multicolumn{3}{|c|}{ Zusammenfassung der Erkenntnisse in Bezug auf nachhaltige Lebensgrundlagen } \\
\hline \multirow[t]{2}{*}{$\begin{array}{l}\text { Armut und } \\
\text { Reichtum }\end{array}$} & $\begin{array}{l}\text { - Leben auf Grönland (Inter- } \\
\text { pretation von Artefakten zu } \\
\text { Ernährung, Handel, Sozial- } \\
\text { struktur) } \\
\text { - Veränderungen der Sozial- } \\
\text { struktur als Folge des Klima- } \\
\text { wandels }\end{array}$ & $\begin{array}{l}\text { - Leben im heutigen Grönland: } \\
\text { Fallstudie Fischerei (Vergleich } \\
\text { von Subsistenzwirtschaft, be- } \\
\text { darfsorientiertem Verkauf, } \\
\text { kommerzieller Fischerei) } \\
\text { - Auswirkungen des Klima- } \\
\text { wandels und Strategien der } \\
\text { Anpassung }\end{array}$ & $\begin{array}{l}\text { - Nachhaltige Fischproduktion } \\
\text { - Strategieentwicklung für die inter- } \\
\text { nationale Zusammenarbeit }\end{array}$ \\
\hline & $\begin{array}{l}\text { Zusammenfassung der Erken } \\
\text { Klimawandel und Global }\end{array}$ & $\begin{array}{l}\text { Intnisse in Bezug auf Armut und } R \in \\
\text { isierung auf die Entwicklung des } V\end{array}$ & $\begin{array}{l}\text { eichtum sowie Auswirkungen von } \\
\text { Verhältnisses zwischen beiden }\end{array}$ \\
\hline \multirow[t]{2}{*}{$\begin{array}{l}\text { Konflikte } \\
\text { und Konflikt- } \\
\text { lösungen }\end{array}$} & $\begin{array}{l}\text { - Besiedlung Grönlands als } \\
\text { Folge von Landkonflikten } \\
\text { - Folgen des Klimawandels } \\
\text { - Auswertung in Bezug auf so- } \\
\text { ziale Konflikte }\end{array}$ & $\begin{array}{l}\text { - Grönland im 20. Jahrhun- } \\
\text { dert bis heute } \\
\text { - lokale Auswirkungen des Kli- } \\
\text { mawandels } \\
\text { - Konflikte um Bergbau (selte- } \\
\text { ne Erden) und Fischerei }\end{array}$ & $\begin{array}{l}\text { - Auswertung in Bezug auf Verbin- } \\
\text { dungen zu Deutschland } \\
\text { - Strategieentwicklung für die inter- } \\
\text { nationale Zusammenarbeit }\end{array}$ \\
\hline & \multicolumn{3}{|c|}{$\begin{array}{l}\text { Zusammenfassung der Erkenntnisse in Bezug auf die Auswirkungen von Klimawandel und } \\
\text { Ressourcennutzung auf gesellschaftliche Konflikte }\end{array}$} \\
\hline
\end{tabular}

das schwierige Verhältnis zwischen wissenschaftlichem Wissen, demokratischer Entscheidung und möglichen Kollateralschäden reflektiert werden.

Aus einem Fundus von Bausteinen lassen sich auf dieser Grundlage fächerübergreifende Module zu den in Tabelle 1 dargestellten Themenfeldern erstellen (Tab. 2). Dabei bietet das Beispiel Grönland als vergleichsweise wenig komplex strukturierte regionale Einheit die Möglichkeit, Zusammenhänge relativ deutlich darzustellen und den Schüler/innen in der Zusammenfassung bewusst zu machen, wie schwierig es ist, selbst in diesen einfachen Strukturen zielführend zu handeln. Jedes Fachthema wird durch Simulationen, Rollenspiele, Weltcafés, die Erstellung von Mind Maps und die Nutzung der Szenariotechnik methodisch herausfordernd aufgearbeitet, sodass die Schülerinnen und Schüler zu kritischem Denken angeregt werden. Zielkonflikte in den Entwicklungsdimensionen werden entsprechend der Lehrplanvorgabe deutlich, was die Notwendigkeit begründeter, demokratischer Entscheidungen unterstreicht.

Der fächerübergreifende Ansatz des Projekts „Expedition Grönland“ geht nicht wie die eingangs beschriebene Variante der Bildung für nachhaltige Entwicklung davon aus, dass der Ansatz der Nachhaltigkeit alle Fächer überflüssig macht und demnächst jede/r alles kann - eine Vision, deren Realität man sich am nur im Themenfeld Nachhaltigkeit (aus)gebildeten Ärztinnen und Ärzten oder Jurist/innen lieber nicht vorstellen möchte. Gleichzeitig unterscheidet er sich aber trotz mancher Übereinstimmungen konzeptuell sowohl vom klassischen, fachorientierten als auch vom verhaltensorientierten Ansatz (Tab. 3). Mit dem klassischen Ansatz gemein hat er die Fokussierung auf das Fach als Basis jeden fächerübergreifenden Unterrichts. Der Geschichtsunterricht trägt dazu bei, den Schülerinnen und Schülern zu helfen, ein Verständnis davon zu erwerben, wie sich Gesellschaften im zeitlichen Ablauf entwickelten. Der Geographieunterricht thematisiert raumbezogene Interaktionen auf unterschiedlichen Maßstabsebenen und die politische Bildung vermittelt demokratische Verfahren zum Ausgleich unterschiedlicher Interessen. Allerdings liegt der Fokus hier nicht wie bei vielen klassischen Varianten auf einem am F1Konzept orientierten starren Faktenwissen, sondern auf einem wissenschaftlichen Wissen, das sich u. a. durch seine dynamische fachliche Systematik auszeichnet. Mit dem verhaltensorientierten Ansatz hat er gemein, dass er sich deutlicher als der klassische fachliche Ansatz auf gesellschaftliche Anforderungen bezieht. Allerdings vermeidet er Zugänge, die dem Indoktrinationsverbot des Beutelsbacher Konsenses widerspre- 
Tab.3: Konzepte fächerübergreifenden Unterrichts im theoretischen Rahmen von Basiskategorien und Bildungsszenarien (eigene Darstellung)

\begin{tabular}{|l|c|l|l|}
\hline \multicolumn{1}{|c|}{ Basiskategorien } & Fachwissen & pädagogische Prozesse & gesellschaftliche Ziele \\
\hline F1-Konzept & $\begin{array}{c}\text { klassischer fächerübergrei- } \\
\text { fender Unterricht }\end{array}$ & & \\
\hline F2-Konzept & fächerübergreifender Unterricht im Projekt „Expedition \\
Grönland” & $\begin{array}{c}\text { verhaltensorientierter fächer- } \\
\text { übergreifender Unterricht }\end{array}$ \\
\hline F3-Konzept & \multicolumn{2}{|r|}{} \\
\hline
\end{tabular}

chen könnten, und sucht dafür Möglichkeiten, die „Fähigkeit, auch anders zu können, als man vermeintlich muss" (Möllers 2009, 115) zu fördern. Allen drei Ansätzen gemeinsam ist, dass sie die pädagogischen Prozesse berücksichtigten. Beim verhaltensorientierten Ansatz ist dies entsprechend der Vorstellungen des F2-Konzepts stärker ausgeprägt als beim klassischen fächerübergreifenden Unterricht. Anders als beim verhaltensorientierten Unterricht bezieht der Ansatz des Grönland-Projekts Fachwissen und Methodik so aufeinander, dass sie eine neue didaktische Wissenseinheit bilden, die sich vom andernorts produzierten akademischen Fachwissen deutlich unterscheidet.

\section{$5 \quad$ Fazit}

Das Projekt „Expedition Grönland“ versteht sich als ein Beitrag zur Bildung für nachhaltige Entwicklung. Mit dem oben dargestellten verhaltensorientierten Ansatz der Bildung für nachhaltige Entwicklung teilt es die Annahme, dass eine Überschreitung von Fachgrenzen notwendig ist, um alltagsrelevante Probleme zu lösen. Allerdings wird diese Annahme theoretisch und praktisch anders bewertet als im verhaltensorientierten Ansatz: Theoretisch wird unterstellt, dass eine fachliche Strukturierung von Wissen aus zwei Gründen sinnvoll ist: Zum einen handelt es sich um ein theoretisch fundiertes Wissen, das in spezialisierten Fachgemeinschaften über oftmals lange Zeiträume entwickelt wurde (vgl. Young 2013). Im Vergleich zum Alltagswissen ist es deswegen systematisch, abstrakt, verlässlich, aber auch dynamisch (vgl. Lambert et al. 2015). Weil es häufig nicht der alltäglichen Intuition entspricht, ist es zum anderen als Korrektiv für Entscheidungen im gesellschaftlichen Raum unerlässlich (vgl. Möllers 2009). Praktisch folgt daraus, dass Fächer und ihr Wissensangebot nicht als nachrangig betrachtet werden, was es mit dem klassischen Ansatz verbindet. Die Fächer sind die Grundlage, auf der fächerübergreifender Unterricht überhaupt erst sinnvoll wird.
Auch hinsichtlich der Frage, welche Art der politischen Bildung mit der Bildung für nachhaltige Entwicklung verfolgt werden soll, unterscheiden sich die beiden Ansätze. Dabei korrespondiert die verhaltensorientierte Variante der Bildung für nachhaltige Entwicklung weitgehend den Vorstellungen von Handlungskompetenz, wie sie in den Bildungsstandards im Fach Geographie für den Mittleren Schulabschluss (DGfG 2007) formuliert sind. Im Vordergrund stehen hierbei weniger Informationshandeln oder politisches Handeln, sondern vor allem das Alltagshandeln. Dieser Schwerpunkt erlaubt es, das Indoktrinationsverbot politischen Unterrichts zwar nicht völlig außer Kraft zu setzen, aber jedenfalls zu relativieren, da die Einflussnahme auf das weniger strittige Feld der moralischen Unterrichtung (vgl. de Haan et al. 2008, 5 i. V.m. 12 f.) verlagert wird. Im Kontext des Projekts „Expedition Grönland“ werden betont keine einfachen Lösungen angeboten, auch keine moralischen. Es folgt vielmehr dem Handlungsbegriff des Faches der politischen Bildung, der seinen Fokus darauflegt, „Meinungen, Überzeugungen und Interessen formulieren, vor anderen angemessen vertreten, Aushandlungsprozesse führen und Kompromisse schließen zu können" (GPJE 2004, 13). Ziel ist somit in erster Linie die Fähigkeit zur rationalen Auseinandersetzung, deren Ergebnis in verantwortungsbewussten Handlungen mündet, wobei diese Handlungen von Mensch zu Mensch verschieden sein können, je nach persönlicher Interessenlage.

Hinsichtlich dieser beiden Aspekte erweisen sich das Projekt „Expedition Grönland“ und der neue Berlin-Brandenburger Lehrplan als kompatibel: Beiden zugrunde liegt ein fachbasierter fächerübergreifender Ansatz. Aber auch hinsichtlich der Bewertung der Handlungskompetenz entspricht das Gesamtwerk des Berlin-Brandenburger Rahmenlehrplans eher dem dargestellten Verständnis des Faches der Politischen Bildung und der Bildung für nachhaltige Entwicklung des Grönlandprojektes als dem Verständnis der Bildungsstandards für den Geographieunterricht und der verhaltensorientierten Bildung für nachhaltige 
Entwicklung. Kommuniziert wird dieses eher politische Verständnis von Handlung dabei durch die explizite Weglassung eines ausgewiesenen, oftmals überaus normativ formulierten Kompetenzbereichs Handlung (SBJW \& MBJS 2015a).

Das Projekt „Expedition Grönland“ repräsentiert somit keinen verhaltensorientierten fächerübergreifenden Unterricht zur Nachhaltigkeit. Es ist allerdings auch nicht schlicht als klassisch fachorientiert zu bezeichnen, denn im Gegensatz zum klassischen Ansatz bezieht es sich dezidiert auf gesellschaftliche Entwicklungen. Es ist weder „unter-sozialisiert" noch „über-sozialisiert" (Young \& Muller 2010), sondern vermittelt zwischen beiden Extremen, indem es den Wert fachwissenschaftlichen Wissens mit der Fähigkeit zur demokratischen Meinungsbildung verbindet.

\section{Literatur}

Arneborg. J., Lynnerup, N. \& Heinemeier, N. (2012): Human Diet and Subsistence Patterns in Norse Greenland AD c. 980-AD c. 1450: Archaeological Interpretations. In: Journal of the North Atlantic, Special Volume 3, 119-133.

Behringer, W. (2011): Kulturgeschichte des Klimas. Von der Eiszeit bis zur globalen Erwärmung. München: dtv.

Berglund, J. (2010): Did the Medieval Norse Society in Greenland Really Fail? In: McAnany, P. A. \& Yoffee, N. (Hrsg.): Questioning Collapse. Human Resilience, Ecological Vulnerability, and the Aftermath of Empire. Cambridge: Cambridge University Press, 45-70.

BMUKK (2012): Die kompetenzorientierte Reifeprüfung aus Geographie und Wirtschaftskunde. Richtlinien und Beispiele für Themenpool und Prüfungsaufgaben. In: GW-Unterricht 125, 100-109.

Butler, J. (2007): Kritik der ethischen Gewalt. Frankfurt am Main: Suhrkamp.

De Haan, G. (2004): Politische Bildung für Nachhaltigkeit. In: Aus Politik und Zeitgeschichte 7-8, 39-46.

De Haan, G., Kamp, G., Lerch, A., Martignon, L., MüllerChrist, G. \& Nutzinger, H. G. (2008): Nachhaltigkeit und Gerechtigkeit. Grundlagen und schulpraktische Konsequenzen. Berlin, Heidelberg: Springer.

DGfG (2007): Bildungsstandards im Fach Geographie für den Mittleren Schulabschluss - mit Aufgabenbeispielen. Bonn: Selbstverlag.

Dugmore, A. J., McGovern, T. H., Vésteinsson, O., Arneborg, J., Streeter, R. \& Keller, C. (2012): Cultural Adaptation, Compounding Vulnerabilities and Conjunctures in Norse Greenland. In: PNAS 109 (10), 3658-3663.

Dugmore, A. J., Keller, C., McGovern, T. H., Casely, A. F. \& Smiarowski, K. (o. J.): Norse Greenland Settlement and Limits to Adaptation. http://uprav.ff.cuni.cz/ ?q=system/files/Dugmore_et_al-Norse_Greenland_Settlement.pdf (04. 01. 2017).
Frei, K. M., Coutu, A. N., Smiarowski, K., Harrison R., Madsen, C. K., Arneborg, J., Frei, R., Guðmundsson, G., Sindbæk, S. M., Woollett, J., Hartman, S., Hicks, M.\& McGovern, T. H. (2015): Was it forwalrus? Viking Age settlement and medieval walrus ivory trade in Iceland and Greenland. In: World Archaeology 47 (3), 439-466.

GPJE(2004):AnforderungenanNationaleBildungsstandards für den Fachunterricht in der Politischen Bildung an Schulen. Ein Entwurf. Schwalbach/Ts.: Wochenschau Verlag.

Huber, L. \& Effe-Stumpf, G. (1994): Der fächerübergreifende Unterricht am Oberstufen-Kolleg. Versuch einer historischen Einordnung. In Krause-Isermann, U., Kupsch, J. \& Schumacher, M. (Hrsg.), Perspektivenwechsel. Beiträge zum fächerübergreifenden Unterricht für junge Erwachsene. Bielefeld: Ambos, 63-86.

Jekel, T. (2008): Plädoyer für relationale Raumkonzepte in einer Bildung für nachhaltige Entwicklung. In: Geographie und ihre Didaktik 36 (2), 65-84.

Klafki, W. (1996): Neue Studien zur Bildungstheorie und Didaktik. Weinheim, Basel: Beltz.

Krings, T. (2002): Zur Kritik des Sahel-Syndromsansatzes aus der Sicht der Politischen Ökologie. In: Geographische Zeitschrift 90 (3-4), 129-141.

Kuckartz, U. (1998): Umweltbewusstsein und Umweltverhalten. Berlin, Heidelberg: Springer.

Labudde, P. (2006): Fachunterricht und fächerübergreifender Unterricht: Grundlagen. In Arnold, K.-H., Sandfuchs, U. \& Wiechmann, J. (Hrsg.): Handbuch Unterricht. Bad Heilbrunn: Klinkhardt, 441-447.

Lambert, D., Solem, M. \& Tani, S. (2015): Achieving Human Potential Through Geography Education: A Capabilities Approach to Curriculum Making in Schools. In: Annals of the Association of American Geographers 105 (4), 723-735.

Lauströer, A. (2008): Bewertungskompetenz durch Bildung für eine nachhaltige Entwicklung. Evaluation einer Unterrichtseinheit zum Thema Massentourismus für die Sekundarstufe I. Saarbrücken: VDM Verlag Dr. Müller.

Luhmann, N. (2002): Das Erziehungssystem der Gesellschaft. Frankfurt am Main: Suhrkamp.

Madsen, C. K. (2014): Pastoral Settlement, Farming, and Hierarchy in Norse Vatnahverfi, South Greenland.Kopenhagen: Dissertation.

Marsden. B. (1997): On Taking the Geography Out of Geographical Education. In: Geography 82 (3), 241-252.

Melde, T. (2012): Nachhaltige Entwicklung durch Semantik, Governance und Management. Zur Selbstregulierung des Wirtschaftssystems zwischen Steuerungsillusionen und Moralzumutungen. Wiesbaden: Springer VS.

Meyer, H. (2007): Grundformen des Unterrichts - Langfassung. Dritte Lektion. Verfügbar unter: http://www.member.uni-oldenburg.de/hilbert.meyer/download/Grundformen_des_Unterrichts_Langfassung.pdf(04.01.2017).

Miossec, A., Arnould, P. \& Veyret, Y. (2004): Développement durable: affaire de tous, approaches de géographes. In: Historiens\&Geographes 387, 85-96. 
Möllers, C. (2009): Demokratie - Zumutungen und Versprechen. Berlin: Wagenbach.

National Museum of Denmark (2008): Resources, Mobility, and Cultural Identity in Norse Greenland AD 980 - 1450. Kopenhagen: National Museum of Denmark.

SBJW \& MBJS (2015a): Rahmenlehrplan Jahrgangsstufen 1-10. Teil A. Bildung und Erziehung in den Jahrgangstufen 1-10. Berlin, Potsdam: Senatsverwaltung für Bildung, Jugend und Wissenschaft Berlin \& Ministerium für Bildung, Jugend und Sport des Landes Brandenburg.

SBJW \& MBJS (2015b): Rahmenlehrplan Jahrgangsstufen 1-10. Teil B. Fachübergreifende Kompetenzentwicklung. Berlin, Potsdam: Senatsverwaltung für Bildung, Jugend und Wissenschaft Berlin \& Ministerium für Bildung, Jugend und Sport des Landes Brandenburg.

SBJW \& MBJS (2015c): Rahmenlehrplan Jahrgangsstufen 1-10. Teil C. Geografie. Jahrgangsstufen 7-10. Berlin, Potsdam: Senatsverwaltung für Bildung, Jugend und Wissenschaft Berlin \& Ministerium für Bildung, Jugend und Sport des Landes Brandenburg.

SBJW \& MBJS (2015d): Rahmenlehrplan Jahrgangsstufen 1-10. Teil C. Geschichte. Jahrgangsstufen 7-10. Berlin, Potsdam: Senatsverwaltung für Bildung, Jugend und Wissenschaft Berlin \& Ministerium für Bildung, Jugend und Sport des Landes Brandenburg.

SBJW \& MBJS (2015e): Rahmenlehrplan Jahrgangsstufen 1-10. Teil C. Politische Bildung. Jahrgangsstufen 7-10. Berlin, Potsdam: Senatsverwaltung für Bildung, Jugend und Wissenschaft Berlin \& Ministerium für Bildung, Jugend und Sport des Landes Brandenburg.

Schindler, J. (2005): Syndromansatz. Ein praktisches Instrument für die Geographiedidaktik. Münster: LIT.

Schnack, J. (2011): Fächerverbindendes Lernen. Von den Grenzen der Fächer und der Lust, sie zu überschreiten. In: Pädagogik 63 (7-8), 6-9.
Stockinger, G. (2013): Archaeologists Find Clues to Viking Mystery. In: SPIEGEL online News International vom 01/10/2013. http://www.spiegel.de/international/zeitgeist/archaeologists-uncover-clues-to-why-vikings-abandoned-greenland-a-876626.html (04. 01. 2017).

Stoltenberg, U. (2006): Innovationsforschung. In Reiß, W. $\&$ Apel, H. (Hrsg.), Bildung für eine nachhaltige Entwicklung. Aktuelle Forschungsfelder und -ansätze. Wiesbaden: VS Verlag für Sozialwissenschaften, 81-86.

Stolz, A. (1991). Die prähistorischen Kulturen Grönlands. In Feest, C. F. (Hrsg.), Am Nordrand der Welt. Eskimo. Schwerpunkt Grönland. Wien: Museum für Völkerkunde.

Uhlenwinkel, A. (2015): Gutachten zum Entwurf des Fachteils C (Geographie) Jahrgangsstufe 7-10 der Anhörungsfassung des Berlin-Brandenburger Lehrplans. https://bildungsserver.berlin-brandenburg.de/fileadmin/ bbb/unterricht/rahmenlehrplaene/Rahmenlehrplanprojekt/anhoerung/materialien/2015-08-21_Gutachten_ Geografie_7-10_Prof._Dr._Uhlenwinkel_HU_Berlin. pdf (04. 01. 2017).

Walk, A. (2002): Im Land der Inuit. Arktisches Tagebuch. Bielefeld: Pendragon.

Wulf, C. (2006): Educación para el Desarrollo Sostenible. Un Plan de Acción Nacional para Alemania. In Wulf, C. \& Newton, B. (Hrsg.), DesarrolloSostenible. Münster: Waxmann, 227-233.

Young, M. (2013): Overcoming the crisis in curriculum theory: a knowledge-based approach. In: Journal of Curriculum Studies 45 (2), 101-118.

Young, M. \& Muller, J. (2010): Three Educational Scenarios for the Future: lessons from the sociology of knowledge. In: European Journal of Education 45 (1), 11-27. 